\title{
Molecular dissection of cardiac repolarization by in vivo Kv4.3 gene transfer
}

\author{
Uta C. Hoppe, Eduardo Marbán, and David C. Johns \\ Institute for Molecular Cardiobiology, The Johns Hopkins University, Baltimore, Maryland 21205, USA \\ Address correspondence to: David C. Johns, Institute for Molecular Cardiobiology, Ross 844, 720 Rutland Avenue, \\ The Johns Hopkins University, Baltimore, Maryland 21205, USA. Phone: (410) 955-2776; Fax: (410) 955-7953; \\ E-mail: djohns@jhmi.edu.
}

Received for publication October 22, 1999, and accepted in revised form March 6, 2000.

\begin{abstract}
Heart failure leads to marked suppression of the $\mathrm{Ca}^{2+}$-independent transient outward current $\left(\mathrm{I}_{\mathrm{to} 1}\right)$, but it is not clear whether $\mathrm{I}_{\mathrm{to} 1}$ downregulation suffices to explain the concomitant action potential prolongation. To investigate the role of $\mathrm{I}_{\mathrm{to} 1}$ in cardiac repolarization while circumventing culturerelated action potential alterations, we injected adenovirus vectors in vivo to overexpress or to suppress $\mathrm{I}_{\text {to1 }}$ in guinea pigs and rats, respectively. Myocytes were isolated 72 hours after intramyocardial injection and stimulation of the ecdysone-inducible vectors with intraperitoneal injection of an ecdysone analog. Kv4.3-infected guinea pig myocytes exhibited robust transient outward currents. Increasing density of $\mathrm{I}_{\text {to1 }}$ progressively depressed the plateau potential in $\mathrm{Kv} 4.3$-infected guinea pig myocytes and abbreviated action potential duration (APD). In vivo infection with a dominant-negative Kv4.3-W362F construct suppressed peak $\mathrm{I}_{\text {to1 }}$ in rat ventriculocytes, elevated the plateau height, significantly prolonged the APD, and resulted in a prolongation by about $30 \%$ of the QT interval in surface electrocardiogram recordings. These results indicate that $\mathrm{I}_{\text {to1 }}$ plays a crucial role in setting the plateau potential and overall APD, supporting a causative role for suppression of this current in the electrophysiological alterations of heart failure. The electrocardiographic findings indicate that somatic gene transfer can be used to create gene-specific animal models of the long QT syndrome.

J. Clin. Invest. 105:1077-1084 (2000).
\end{abstract}

\section{Introduction}

Congestive heart failure is a common and highly lethal cardiovascular disorder. Despite various therapeutic interventions that have decreased overall mortality in recent years, patients with heart failure remain at high risk of sudden cardiac death (1-4). Myocytes from humans and from various animal models of heart failure exhibit characteristic changes in the currents that shape the action potential, notably a reduction of the calcium-independent transient outward current $\left(\mathrm{I}_{\mathrm{to} 1}\right)$ (5-7). Nevertheless, the role of $I_{\text {to } 1}$ in shaping the action potential remains unclear, making it difficult to assess whether this change is adaptive, maladaptive, or simply a marker of the disease process.

In most species studied to date, $\mathrm{I}_{\mathrm{to1}}$ is encoded either by $\mathrm{Kv} 4.2$ or $\mathrm{Kv} 4.3$, or by a combination of the 2 (8-12). To probe the role of Kv4.x channels in cardiac electrophysiology, we previously suppressed $\mathrm{I}_{\mathrm{to} 1}$ in primary cultured cardiomyocytes using an adenovirus that encoded a truncated, dominant-negative form of Kv4.2 (10). However, further elucidation of the contribution of $\mathrm{I}_{\text {to } 1}$ to the action potential was complicated by culture-induced changes in the control action potential (13). To circumvent culture-related effects on the action potential waveform, we previously used cell fusion to introduce premade functional $\mathrm{Kv} 4.3$ ion channels into freshly isolated guinea pig ventricular myocytes (14), which lack an endogenous $\mathrm{I}_{\text {to1 }}(15)$.
Introduction of $\mathrm{I}_{\mathrm{to} 1}$ into these acutely dissociated cardiomyocytes accelerated early repolarization, depressed the action potential plateau and abbreviated APD. However, cell fusion is not well-suited for dominantnegative channel suppression or for in vivo use.

In the present study, we delivered ion channel constructs by direct intramyocardial injection of recombinant adenovirus vectors in vivo and studied the electrophysiology of cardiomyocytes freshly isolated from such hearts. Previously, we had shown the utility of the ecdysone inducible system in expressing ion channels (16); here, we have modified that system to function more efficiently in vivo. The results indicate that $\mathrm{I}_{\mathrm{to} 1}$ either directly or indirectly by its influence on the early repolarization process plays a prominent role in setting the plateau potential and APD.

\section{Methods}

The present investigation conforms to the standards of the US National Institutes of Health regarding care and use of laboratory animals, and was performed in accordance with the guidelines of the Animal Care and Use Committee of the Johns Hopkins University.

Plasmid construction and adenovirus preparation. The plasmids pC8I and pGFPKir2.1-AAA and the adenovirus shuttle vectors pAdCGI, pAdCGI-DBEcR, pAdVgRXR, and PAdEGI have been described elsewhere $(10,14,16,17)$. The coding sequence from rat Kv4.3 
(kindly supplied by B. Rudy, New York University, New York, New York, USA) was cloned into the multiple cloning site of pAdEGI, to generate pAdEGIKv4.3. In accordance with a previously reported dominant-negative Kv4.2 mutation (18), the point mutation W362F was introduced into Kv4.3 by sitedirected mutagenesis, creating the vector pAdEGIKv4.3W362F. The coding sequence of human CD8 was cut out of pC8I and cloned into pAdEGI-Kv4.3 and pAdEGI-Kv4.3W362F in place of the EGFP sequence, to make pAdE8I-Kv4.3 and pAdE8IKv4.3W362F, respectively. The coding sequence of the hybrid ecdysone receptor DBEcR from pAdCGIDBEcR was cloned into pAdCGI in place of the EGFP sequence, to generate pAdC-DBEcR.

Adenovirus vectors were generated by Cre-lox recombination of purified $\psi 5$ viral DNA and shuttle vector DNA as described previously $(16,19)$. The recombinant products were plaque purified, expanded, and purified on $\mathrm{CsCl}$ gradients yielding concentrations on the order of $10^{10}$ plaque-forming units (PFUs) per milliliter.

Transient transfections. Twenty-four hours before transfection, CHO-K1 cells (ATCC CCL 61; American Type Culture Collection, Manassas, Virginia, USA) were seeded at a density of $2.0 \times 10^{5} / 35 \mathrm{~mm}$. Cells were transfected with plasmid DNA $(1 \mu \mathrm{g} /$ well total $)$ using Lipofectamine Plus (Life Technologies Inc., Gaithersburg, Maryland, USA) as directed by the manufacturer. After 4 hours, transfection media was replaced with normal growth media. Expression was induced by addition of ponasterone A $10 \mu \mathrm{M}$ (Invitrogen Corp., San Diego, California, USA) for 72 hours.

Animals. Adult rats (Sprague-Dawley; 200-250 g) and adult guinea pigs $(200-250 \mathrm{~g})$ underwent direct intramyocardial adenovirus injection using a 30-gauge needle as reported previously $(17,20)$. For patch-clamp experiments, the anterior wall of the left ventricle was infiltrated under direct viewing 3-5 times with a total volume of $150 \mu \mathrm{L}$ of an adenovirus mixture containing approximately $5 \times 10^{8} \mathrm{PFU}$ AdCGI-DBEcR with saline (controls), approximately $5 \times 10^{8}$ PFU AdE8I-Kv4.3 (guinea pigs), or approximately $5 \times 10^{8}$ PFU AdE8IKv4.3W362F (rats). For electrocardiogram (ECG) recordings, the left ventricle of rats was injected more widespread at multiple sites ( 10 times) from the basis to the apex of the anterior, lateral, and posterior wall, with a total volume of $220 \mu \mathrm{L}$ of an adenovirus mixture containing approximately $8 \times 10^{8}$ PFU AdC-DBEcR with approximately $8 \times 10^{8} \mathrm{PFU}$ AdE8I-Kv4.3W362F or approximately $8 \times 10^{8} \mathrm{PFU}$ AdEGI (controls). After the chest was closed, animals were injected intraperitoneally with $45 \mathrm{mg}$ of the nonsteroidal ecdysone receptor agonist, GS-E ([N-(3-methoxy-2-ethylbenzoyl)$N^{\prime}$-(3,5-dimethylbenzoyl)- $N^{\prime}$-tert-butylhydrazine]; kindly provided by Rohm and Haas Co., Spring House, Pennsylvania, USA), dissolved in $90 \mu \mathrm{L}$ DMSO and 360 $\mu \mathrm{L}$ sesame oil. Ecdysone hormones are not known to affect mammalian physiology. GS-E is a member of the bisacylhydrazine chemical family that have been shown to have no adverse effects over a broad dosage range in mammals (21).

Myocyte isolation and electrophysiology. Seventy-two hours after intramyocardial injection, guinea pig and rat left ventricular myocytes were isolated as described previously $(14,22)$. The yield of infected myocytes was $1-3 \%$. Experiments were carried out using the wholecell patch-clamp technique (23) with an Axopatch 200B amplifier (Axon Instruments, Foster City, California, USA) while sampling at $10 \mathrm{kHz}$ and filtering at $2 \mathrm{kHz}$. All myocyte recordings were performed at a temperature of $37^{\circ} \mathrm{C}$, whereas $\mathrm{CHO}-\mathrm{K} 1$ cell experiments were performed at $20^{\circ} \mathrm{C}$.

Cells were superfused with a physiological saline solution containing $138 \mathrm{mmol} / \mathrm{L} \mathrm{NaCl}, 5 \mathrm{mmol} / \mathrm{L} \mathrm{KCl}, 2$ $\mathrm{mmol} / \mathrm{L} \mathrm{CaCl}_{2}, 10 \mathrm{mmol} / \mathrm{L}$ glucose, $0.5 \mathrm{mmol} / \mathrm{L} \mathrm{MgCl}_{2}$, $10 \mathrm{mmol} / \mathrm{L}$ HEPES; $\mathrm{pH}$ was adjusted to 7.4 with $\mathrm{NaOH}$. For $\mathrm{I}_{\mathrm{Kr}}$ recordings, $500 \mu \mathrm{mol} / \mathrm{L} \mathrm{BaCl}_{2}$ and $200 \mu \mathrm{mol} / \mathrm{L}$ $\mathrm{CdCl}_{2}$ were added to block $\mathrm{I}_{\mathrm{K} 1}$ and $\mathrm{I}_{\mathrm{CaL}}$, respectively. For $\mathrm{I}_{\mathrm{CaL}}$ recordings, $\mathrm{KCl}$ was substituted with an equal amount of $\mathrm{NaCl}$. The micropipette electrode solution was composed of $130 \mathrm{mmol} / \mathrm{L} \mathrm{K}$-glutamate, $9 \mathrm{mmol} / \mathrm{L}$ $\mathrm{KCl}, 8 \mathrm{mmol} / \mathrm{L} \mathrm{NaCl}, 0.5 \mathrm{mmol} / \mathrm{L} \mathrm{MgCl} 2,10 \mathrm{mmol} / \mathrm{L}$ HEPES, $2 \mathrm{mmol} / \mathrm{L}$ EGTA, and $5 \mathrm{mmol} / \mathrm{L} \mathrm{Mg-ATP;} \mathrm{pH}$ was adjusted to 7.2 with $\mathrm{KOH}$. L-type calcium currents were measured with an internal solution of $40 \mathrm{mmol} / \mathrm{L}$ $\mathrm{CsCl}$ 140, $10 \mathrm{mmol} / \mathrm{L}$ HEPES, $2 \mathrm{mmol} / \mathrm{L}$ EGTA, and 5 $\mathrm{mmol} / \mathrm{L} \mathrm{Mg-ATP}$ ( $\mathrm{pH}$ 7.2). Borosilicate microelectrodes had tip resistances of 1-4 M $\Omega$ when filled with the internal recording solution. Data were corrected for the measured liquid junction potential $(-18 \mathrm{mV})(24)$.

Voltage clamp experiments were performed with an interepisode interval of 2 seconds. Action potentials were initiated by short depolarizing current pulses (2-3 ms, 500-800 pA) at $2 \mathrm{~Hz}$. APD was measured as the time from the overshoot to the indicated percentage of repolarization. A xenon arc lamp was used to view GFP and R-phycoerythrin-conjugated CD8 antibody (Sigma Chemical Co., St. Louis, Missouri, USA) at $488 / 530 \mathrm{~nm}$ and $543 / 605$, respectively (excitation/emission).

ECGs. Surface ECGs were recorded immediately after operation and 72 hours after intramyocardial injection. Rats were anesthetized with metafane, and needle electrodes were placed under the skin. Similar to ECGs in transgenic mice (18), electrode positions were optimized to obtain maximal amplitude recordings, enabling accurate measurements of QT intervals. ECGs were simultaneously recorded from standard lead II, modified lead I with the arm electrode placed at the base of the sternum, and modified lead III with the arm electrode placed to the back of the left shoulder. Needle electrode positions were marked postoperatively on the rats' skin to ensure exactly the same electrode position for 72 -hour controls. Signals were digitized at $2 \mathrm{kHz}$.

Confocal imaging. Images were taken on a laser confocal microscope (PCM 2000; Nikon Inc., Melville, New York, USA) with a $60 \times$ water immersion objective lens. GFP was imaged with an argon laser at 488/520 \pm 15 
$\mathrm{nm}$, R-phycoerythrin-conjugated CD8 antibody with a neon laser at 543/605 $\pm 16 \mathrm{~nm}$ (excitation/emission). Statistical analysis. Pooled data are presented as mean \pm SEM. Regression analysis was used to test for a relationship between $\mathrm{I}_{\mathrm{to} 1}$ density and repolarization velocity, plateau height, and APD. Comparisons between groups were performed using 1-way ANOVA. $P$ values less than 0.05 were deemed significant.

\section{Results}

Overexpression of $I_{\text {to1 }}$ in guinea pig myocytes. We used a novel hybrid Drosophila/Bombyx ecdysone receptor (DB$\mathrm{EcR})$ that effectively mediates inducible transgene expression in vivo (17). Adenoviral DB-EcR vectors demonstrated a greater than 40 -fold increase of luciferase activity in myocardial tissue in vivo upon stimulation with the chemical ecdysone analog GS-E. To verify that our hybrid ecdysone system was sufficient not only to express a sensitive reporter gene like luciferase but also functional membrane proteins like ion channels, we first sought to produce effective overexpression of wild-type ion channels. We chose guinea pigs to introduce $\mathrm{I}_{\mathrm{to} 1}$ into myocardial tissue, as guinea pig ventriculocytes lack $\mathrm{I}_{\mathrm{to} 1}$ under physiological conditions (15). This enabled us to evaluate the efficiency of inducible ion channel expression and to compare resulting effects of $\mathrm{I}_{\mathrm{to} 1}$ overexpression on the guinea pig action potential with previously observed changes using cell fusion to introduce $\mathrm{I}_{\text {to1 }}$ (14).

Before attempting to modify the electrophysiology of guinea pig myocytes, we needed to prove that adenoviral infection itself did not alter the basic electrophysiology. Guinea pig myocardium was injected with the receptor virus AdCGI-DBEcR alone or a reporter virus AdCGI, and myocytes were isolated 72 hours later. Infected cells were identified by their obvious green fluorescence. To test for nonspecific effects of adenovirus infection, action potentials, L-type calcium currents $\mathrm{I}_{\mathrm{CaL}}$, inward rectifier currents $\mathrm{I}_{\mathrm{K} 1}$, and delayed rectifier currents $\mathrm{I}_{\mathrm{Kr}}$ were recorded in infected cells and compared with uninfected myocytes from the same hearts. Adenovirus infection did not produce any appreciable effects on the waveform and duration of action potentials or on current size of $\mathrm{I}_{\mathrm{CaL}}, \mathrm{I}_{\mathrm{K} 1}$, and $\mathrm{I}_{\mathrm{Kr}}$. Mean resting membrane potential $(-88.1 \pm 1.2$ vs. $-87.8 \pm 1.3 \mathrm{mV})$, overshoot ( $38.6 \pm 2.6$ vs. $39.2 \pm 2.2 \mathrm{mV})$, and APDs measured at $50 \%(119.6 \pm 14.8$ vs. $127.4 \pm 23.2 \mathrm{~ms})$ and $90 \%(142.5 \pm 10.2$ vs. $138.7 \pm 26.7 \mathrm{~ms})$ repolarization did not differ significantly in noninfected $(n=6)$ and infected $(n=5)$ myocytes, respectively. As shown in Figure 1 , peak $\mathrm{I}_{\mathrm{CaL}}$ current density at $0 \mathrm{mV}$ after a prepulse to $-40 \mathrm{mV}, \mathrm{I}_{\mathrm{Kr}}$ tail current density at $-100 \mathrm{mV}$ after a depolarization step to $-10 \mathrm{mV}$ for $200 \mathrm{~ms}$, and $\mathrm{I}_{\mathrm{K} 1}$ current density measured at the end of 500-ms hyperpolarization step to $-100 \mathrm{mV}(-6.8 \pm 2.3[n=10]$ vs. $6.2 \pm$ $0.5 \mathrm{pA} / \mathrm{pF}[n=18])$ were similar in noninfected and infected myocytes, respectively. We have previously shown that adenovirus infection itself does not affect $\mathrm{I}_{\mathrm{to} 1}$ in rat cardiomyocytes (25).

To overexpress $\mathrm{I}_{\mathrm{to} 1}$, guinea pig myocardium was coinjected with the receptor virus AdCGI-DBEcR and the reporter virus AdE8I-Kv4.3, which drives the separate translation of the surface antigen CD8 and the ion channel Kv4.3 under control of a single modified ecdysone promoter (26). Seventy-two hours after injection and stimulation with GS-E, myocytes were isolated and CD8 was viewed with monoclonal R-phycoerythrin-conjugated CD8 antibodies. Figure 2 shows a confocal image of a typical coinfected myocyte. The cytosolic green fluorescence of the myocyte verifies infection with the receptor virus, and the red staining of the surface membrane indicates expression of CD8 from the second virus, which also carries the ion channel gene. Infected cells were readily distinguishable

\section{Figure 1}

L-type calcium currents $I_{\mathrm{Ca}}$ and delayed rectifier currents $I_{\mathrm{Kr}}$ were not different in noninfected guinea pig myocytes compared with myocytes that were in vivo infected with a reporter (GFP) adenovirus. Peak $I_{\text {CaL }}$ current density was measured at $0 \mathrm{mV}$ after a prepulse to $-40 \mathrm{mV}$ in noninfected (a) and GFPinfected (b) myocytes. $I_{\mathrm{Kr}}$ tail currents in noninfected cells (d) measured at -100 $\mathrm{mV}$ after a 200-ms depolarization step to $-10 \mathrm{mV}$ were compared with GFPinfected myocytes (e). Original current traces and mean current densities indicate that adenovirus infection itself did not affect $I_{\mathrm{CaL}}(\mathbf{a}-\mathbf{c})$ and $I_{\mathrm{Kr}}(\mathbf{d}-\mathbf{f})$. a

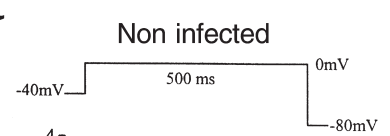

b

d
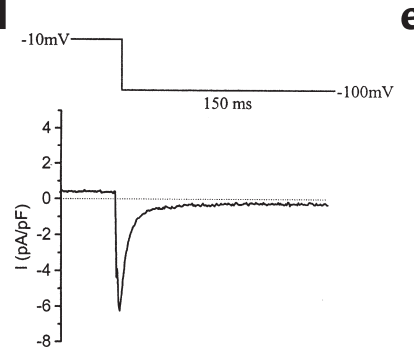

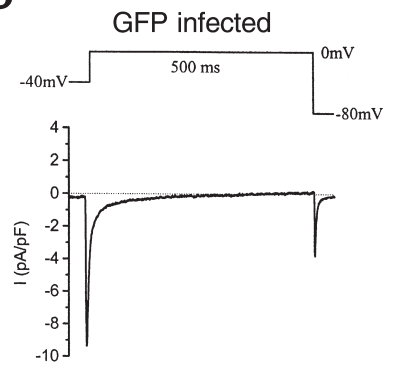

C

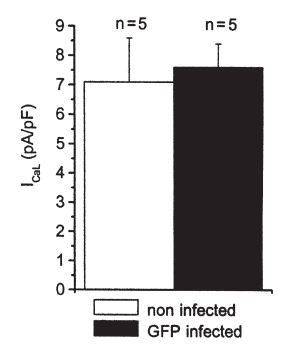

f

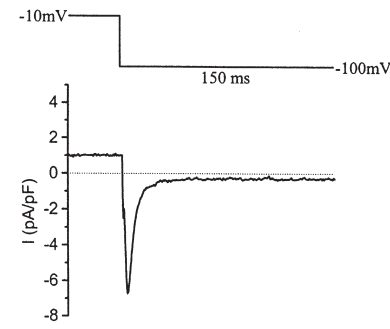

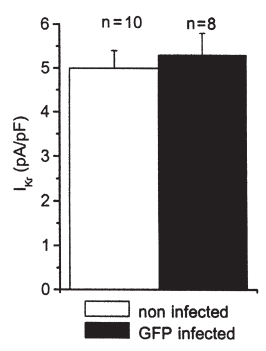


from the background autofluorescence of noninfected cells. Most (>95 \%) of AdCGI-DBEcR infected (green) cells also expressed CD8, indicating that coinfection of the 2 viruses is the rule rather than the exception.

Myocytes infected with Kv4.3 in vivo exhibited robust transient outward currents. The density of $\mathrm{I}_{\text {to1 }}$ was $2.0-52.9 \mathrm{pA} / \mathrm{pF}$ at $+40 \mathrm{mV}$. Mean peak current density was $22.0 \pm 5.8 \mathrm{pA} / \mathrm{pF}$ at $+40 \mathrm{mV}(n=10)$. Figure 3 shows transient outward currents and corresponding action potentials recorded in 4 different $\mathrm{Kv} 4.3$-infected myocytes that exhibited different $\mathrm{I}_{\text {to1 }}$ amplitudes. In vivo adenovirus-mediated introduction of $\mathrm{I}_{\mathrm{to} 1}$ substantially changed the action potential waveform of freshly isolated guinea pig myocytes. The modification of the action potential became more pronounced as the amount of $\mathrm{I}_{\mathrm{to} 1}$ increased. Extremely large $\mathrm{I}_{\mathrm{to} 1}$ densities resulted in a spikelike configuration of the action potential reminiscent of that recorded in normal rat ventriculocytes (Figure 3d). These changes in the action potential waveform after in vivo $\mathrm{I}_{\mathrm{to} 1}$ expression were virtually identical to modifications of the action potential induced by fusion of guinea pig myocytes with Kv4.3expressing $\mathrm{CHO}$ cells (14).

$\mathrm{I}_{\mathrm{to} 1}$ accelerated the initial repolarization velocity of Kv4.3-infected guinea pig myocytes. Initial repolarization velocity measured $3 \mathrm{~ms}$ after the overshoot became progressively faster with increasing $\mathrm{I}_{\text {to1 }}$ density $(r=$ $-0.82 ; P=0.003 ; n=10)$. Among all action potential parameters, the plateau potential (i.e., the voltage at $\mathrm{d}^{2} \mathrm{~V} / \mathrm{dt}^{2}=0$; ref. 27) correlated best with $\mathrm{I}_{\mathrm{to1}}$ density $(r=$ $-0.97 ; P<0.0001 ; n=10$ ) (Figure 4 ). Introduction of $\mathrm{I}_{\mathrm{to} 1}$ generally depressed the whole plateau phase; only in 1

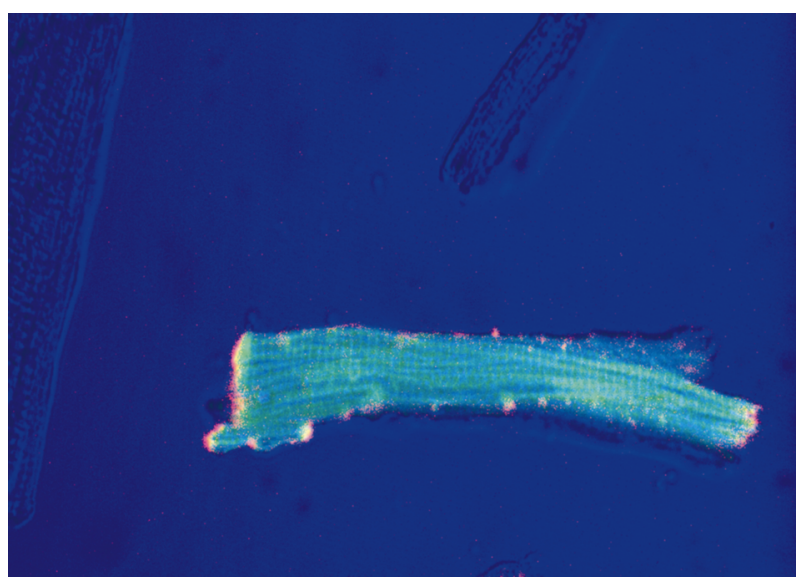

\section{Figure 2}

Confocal fluorescence image of typical in vivo coinfected guinea pig myocytes. Guinea pig myocardium was coinjected with the receptor virus AdCGI-DBEcR and the reporter virus AdE8I-Kv4.3. Seventy-two hours after injection and stimulation with GS-E, myocytes were isolated and CD8 was viewed with R-phycoerythrin-conjugated CD8 antibodies. The cytosolic green fluorescence of the myocyte verifies infection with the receptor virus. Red staining of the surface membrane indicates induced expression of CD8 from the reporter virus, which also carries the ion channel gene. Infected cells were readily distinguishable from the background autofluorescence of noninfected cells (transmitted light image shown in blue plane). cell was a notch-and-dome configuration obtained. $\mathrm{I}_{\text {to1 }}$ also decreased the overall $\mathrm{APD}$ both at $50 \%\left(\mathrm{APD}_{50} ; r=\right.$ $-0.71 ; P=0.02)$ and $90 \%\left(\mathrm{APD}_{90} ; r=-0.70 ; P=0.02\right)$ repolarization in an $\mathrm{I}_{\mathrm{to} 1}$ density-dependent manner.

$I_{\text {to1 }}$ knockout in rat myocytes. A unique advantage of gene transfer over cell fusion is the ability to use dominant-negative strategies to achieve suppression of selected ion channel families. Because the major part of $\mathrm{I}_{\mathrm{to} 1}$ is encoded by Kv4.2 and/or Kv4.3 in most species (8, $10,18)$, we introduced a point mutation into $\mathrm{Kv} 4.3$ (W362F) to design a dominant-negative Kv4.x construct for in vivo $I_{\text {to } 1}$ knockout. The corresponding mutation in Kv4.2 has previously been reported to exhibit dominant-negative function in transgenic mice, without evidence of cytotoxicity (18). Before expressing Kv4.3-W362F in vivo, we confirmed its dominant-negative efficacy in cotransfection experiments. CHO-K1 cells cotransfected with $\mathrm{Kv} 4.3$ wild-type and $\mathrm{Kv} 4.3$ W362F exhibited a $\mathrm{I}_{\mathrm{to1}}$ density of $75.6 \pm 4.9 \mathrm{pA} / \mathrm{pF}(n=$ 17) at $+40 \mathrm{mV}$ compared with $167.0 \pm 7.0 \mathrm{pA} / \mathrm{pF}(n=$ 13 ) in CHO-K1 cells cotransfected with Kv4.3 wild-type and an unrelated Kir2.1 construct carrying a pore mutation (Kir2.1-AAA) $(10)(P<0.05)$. CHO-K1 cells cotransfected with Kv4.3-W362F and Kir2.1-AAA had no detectable transient outward current $(n=5)$.

To probe the effect of $\mathrm{I}_{\mathrm{to} 1}$ knockout on the action potential of freshly isolated rat myocytes, we coinjected the left ventricular anterior wall of rats with AdCGIDBEcR and AdE8I-Kv4.3W362F. As in the guinea pig experiments already described here, myocytes were isolated 72 hours after infection and induction with GSE, CD8 was viewed by antibodies, and infected myocytes were identified by their green fluorescence and red surface membrane staining.

Figure 5 shows the fully primed and prepulse-inactivated $(-100 \mathrm{mV}$ and $0 \mathrm{mV}$ holding potentials, respectively) transient outward currents elicited by test pulses to $+40 \mathrm{mV}$ in a noninfected myocyte (Figure $5 \mathrm{a}$ ) and in another myocyte infected with AdE8I-Kv4.3W362F (Figure $5 \mathrm{~b}$ ). The pooled data for the peak outward currents $(P=0.002$; Figure $5 c)$ and the difference between the fully primed and prepulse-inactivated currents $(P=$ 0.0004; Figure 5d) confirmed the significant suppression of native rat cardiac $\mathrm{I}_{\mathrm{to} 1}$ by in vivo infection with Kv4.3-W362F $(n=10$ noninfected and 7 infected myocytes). Similar to the previously reported in vitro knockout of $\mathrm{I}_{\text {to1 }}$ using a truncated Kv4.2 construct (10), the suppression of outward current was substantial but not complete, presumably as a result of remaining competitive expression of wild-type channels.

In addition to $\mathrm{I}_{\mathrm{to} 1}$ reduction, we observed a lesser reduction of the maintained outward current measured at the end of a 500-ms depolarization pulse to

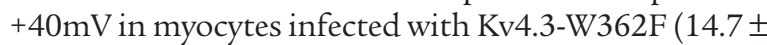
$2.2 \mathrm{pA} / \mathrm{pF} ; n=7)$ compared with noninfected cells $(22.2$ $\pm 1.8 \mathrm{pA} / \mathrm{pF} ; n=10)(P=0.01)$. This suppression of the maintained component may be due to a knockout of native Kv4.1 that is expressed at low levels in rat ventricular myocardium (8) and/or to incomplete inacti- 


\section{Figure 3}

Transient outward currents and action potentials of 4 different guinea pig myocytes (a-d) that were infected in vivo with AdCGI-DBEcR and AdE8I-Kv4.3. Seventy-two hours after injection and stimulation with GS-E, myocytes were isolated. Transient outward currents were elicited by depolarization pulses to $+40 \mathrm{mV}$ from a holding potential of -100 $\mathrm{mV}$. In vivo adenovirus-mediated introduction of $I_{\text {to1 }}$ substantially changed the action potential waveform of acutely dissected guinea pig myocytes in an $I_{\text {to } 1}$ density-dependent manner, resulting in a depression of the plateau voltage and abbreviation of the overall APD. (d) Introduction of very large $I_{\text {to } 1}$ densities caused a spikelike action potential. a
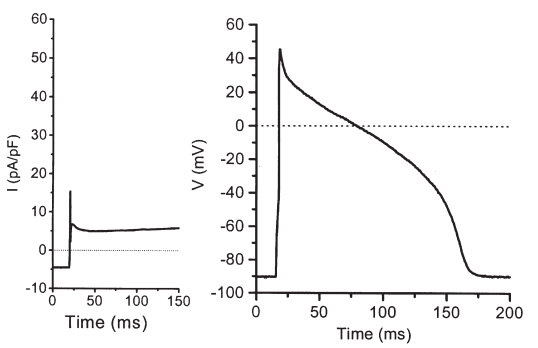

C
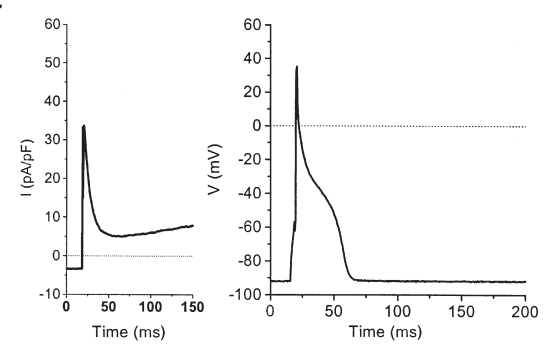

b
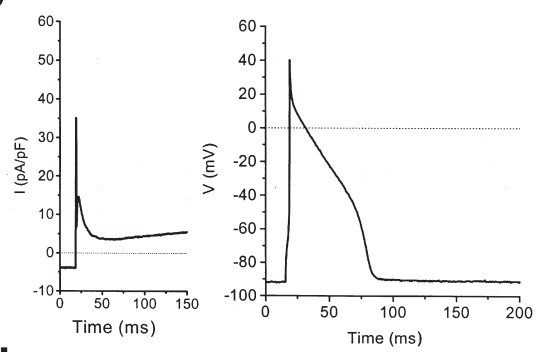

d
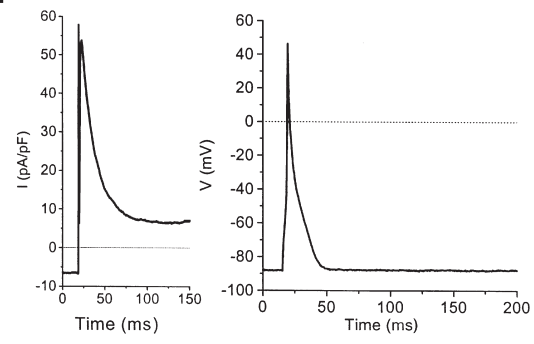

vation of $\mathrm{Kv} 4.2$ at the end of the depolarization pulse (28). We did not find any differences in cell size (78.5 \pm 7.6 vs. $85.5 \pm 8.9 \mathrm{pF}$ ), resting membrane potential $(-86.4 \pm 0.6$ vs. $-86.6 \pm 1.5 \mathrm{mV})$, inward current density at $-100 \mathrm{mV}(-4.4 \pm 0.8$ vs. $-3.7 \pm 0.3 \mathrm{pA} / \mathrm{pF})$, or action potential overshoot $(25.4 \pm 2.4$ vs. $28.7 \pm 4.2 \mathrm{mV})$ between noninfected $(n=10)$ and Kv4.3W362F-infected $(n=7)$ myocytes, respectively.

Action potential recordings from Kv4.3W362Finfected myocytes demonstrated the physiological consequences of $I_{\text {to1 }}$ suppression on the repolarization of freshly isolated rat cardiomyocytes (Figure 6). Knockout of $\mathrm{I}_{\mathrm{to} 1}$ substantially changed the action potential waveform of infected cells (Figure 6, $c$ and d) compared with noninfected myocytes (Figure 6, a and b), resulting in a more pronounced, elevated plateau phase and a prolongation of the overall $\mathrm{APD}$. In 1 rat myocyte, $\mathrm{I}_{\mathrm{to} 1}$ reduction led to a notch-and-dome action potential waveform (Figure 6d). In this myocyte, frequent early after depolarizations (EADs) were recorded (Figure 6e); such events were never observed in wild-type rat myocytes. To quantify further the effect of $\mathrm{I}_{\text {to1 }}$ suppression on the APD, we plotted the mean APDs of wild-type $(n=10)$ and $\mathrm{Kv} 4.3$-W362F-infected $(n=7)$ myocytes as a function of the percentage of repolarization (Figure 6f). $\mathrm{I}_{\mathrm{to} 1}$ suppression did not exhibit a significant effect on the APD at 10-50\% repolarization, but significantly prolonged later values of APD (e.g., $\mathrm{APD}_{90}, P=0.0001$ ).

Localized injection of the left anterior ventricular wall resulted in no ECG differences between GFP-infected rats and those infected with the Kv4.3 dominant-negative construct ( $n=3$ in each group; data not shown). To determine whether a more widespread Kv4.x suppression in rats could affect the surface ECG, adenovirus vectors were injected at multiple sites into the left ventricular myocardium targeting $60-80 \%$ of the left ventricular free wall. Although the QT interval has been proposed to be heart-rate independent in rats $(29,30)$, QT meas- urements were performed at similar heart rates postoperatively and 72 hours after infection in individual animals. ECGs revealed a $29.8 \pm 6.0 \%$ prolongation of the QT interval in Kv4.3-W362F-infected rats 72 hours after intramyocardial injection $(117.7 \pm 6.8 \mathrm{~ms}$, RR interval $389.8 \pm 10.1 \mathrm{~ms}$ ) compared with immediate postoperative recordings $(90.5 \pm 1.3 \mathrm{~ms}$, $\mathrm{RR}$ interval $387.7 \pm 11.4$ $\mathrm{ms})(P=0.02 ; n=3)$ (Figure $7, \mathrm{c}$ and $\mathrm{d})$. This is consistent with the marked prolongation of ventricular action potentials that was seen in isolated cells. No change in the QT interval was observed in GFP-infected control animals $(93.8 \pm 2.5 \mathrm{~ms}$, RR interval $390.0 \pm 2.7 \mathrm{~ms}$ vs. $92.9 \pm 2.9 \mathrm{~ms}$, RR interval $398.5 \pm 10.3 \mathrm{~ms}$ postoperatively and 72 hours after infection, respectively; $n=3$ ) (Figure 7, a and b). During the 72-hour postoperative period, survival of the Kv4.3-W362F-injected animals was not different from controls.
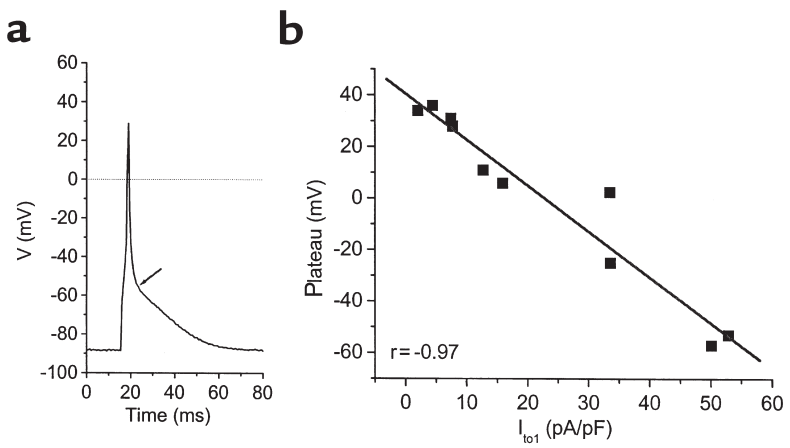

\section{Figure 4}

Effect of $I_{\text {to1 }}$ current size on the height of the action potential plateau in guinea pig myocytes infected in vivo with AdCGIDBEcR and AdE8I-Kv4.3. (a) Introduction of $\mathrm{I}_{\text {to } 1}(50.1 \mathrm{pA} / \mathrm{pF})$ into a guinea pig myocyte depressed the voltage of the early plateau phase measured at $\mathrm{d}^{2} \mathrm{~V} / \mathrm{dt}^{2}=0$ (arrow) (at the transition from early repolarization to final repolarization; ref. 27). (b) The suppression of the action potential plateau correlated well with the introduced $\mathrm{I}_{\mathrm{to} 1}$ density $(n=10)$. 


\section{Discussion}

The present study demonstrates that $\mathrm{I}_{\mathrm{to1}}$ plays an important role in setting the plateau potential and APD. In vivo adenovirus-mediated overexpression of $\mathrm{I}_{\mathrm{to} 1}$ in guinea pig myocytes abbreviates action potentials; conversely, dominant-negative suppression of $\mathrm{I}_{\mathrm{too}}$ in rat cardiocytes delays repolarization. Previously, we used cell fusion to introduce Kv4.3 into freshly isolated guinea pig cells to probe the contribution of $\mathrm{I}_{\mathrm{to1}}$ to cardiac action potentials (14). Cell fusion enabled us to circumvent the changes of excitability that complicate in vitro adenovirus-mediated ion channel expression in primary culture (10). Besides overexpressing $\mathrm{I}_{\mathrm{to} 1}$ in myocytes that physiologically lack $\mathrm{I}_{\mathrm{to} 1}(15)$, we sought to probe the role of $\mathrm{I}_{\mathrm{to} 1}$ in the action potential of cells that express endogenous transient outward currents, especially because $I_{\text {to1 }}$ reduction is the salient electrical feature of heart failure myocytes (5-7). However, cell fusion is not suited for dominant-negative channel suppression, as functional current knockout requires formation of new channel complexes that is not believed to occur in the surface membrane itself but rather in subcellular compartments (31). Besides dominant-negative suppression of $\mathrm{I}_{\mathrm{to}}$ in primary cultured myocytes (10) and pharmacological block $(6,32)$, reduction of $I_{\text {to1 }}$ has been previously achieved by the generation of transgenic mice (18). However, transgene expression that is not under control of a regulated expression system may lead to adaptive changes of other genes in transgenic animals during development, which resulted in upregulation of another noninactivating outward current in Kv4.2 knockout mice (18). Additionally, creation of transgenic animals is time consuming and limited in its species versatility. The
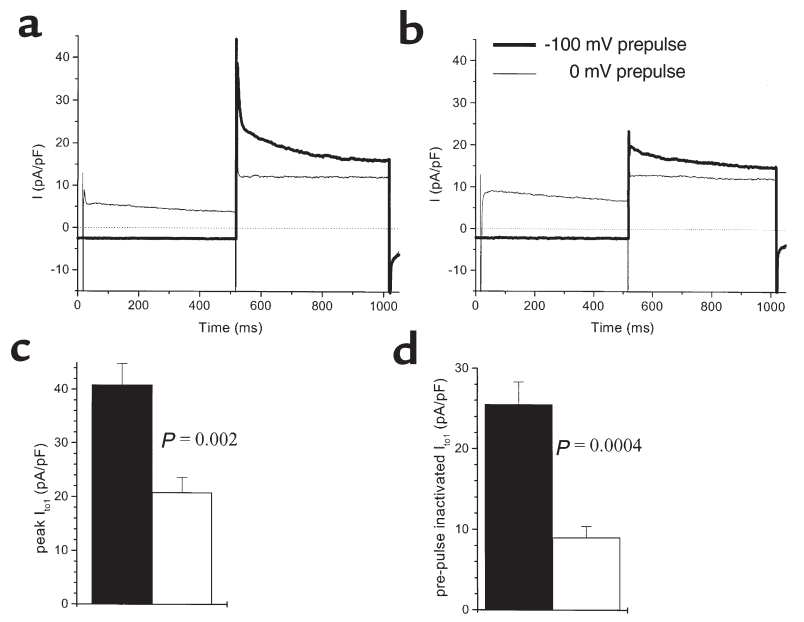

Figure 5

In vivo expression of AdE8I-Kv4.3-W362F suppressed $\mathrm{I}_{\text {to1 }}$ in adult rat myocytes. Transient outward currents elicited by test pulses to +40 $\mathrm{mV}$ in noninfected myocytes (a) are compared with currents in AdE8I-Kv4.3-W362F-infected myocytes (b). Mean values of peak I to1 (c) and of the difference between the fully primed and prepulse-inactivated $(0 \mathrm{mV})(\mathbf{d})$ currents indicate a significant suppression of native rat cardiac $\mathrm{I}_{\text {to } 1}$ in $\mathrm{Kv} 4.3-\mathrm{W} 362 \mathrm{~F}$-infected myocytes ( $n=7$; filled bars) compared with wild-type ( $n=10$; shaded bars). use of recombinant adenovirus vectors for in vivo transgene expression in adult animals enabled us to elucidate the effect of $\mathrm{I}_{\mathrm{to1}}$ overexpression and of dominant-negative $\mathrm{I}_{\mathrm{to} 1}$ suppression while obviating nonspecific drug effects, culture-related alterations of the action potential, and long-term compensatory channel upregulation.

Action potential recordings both in rat myocytes after $\mathrm{I}_{\text {to } 1}$ knockout and in guinea pig cells after $\mathrm{I}_{\text {to1 }}$ overexpression by in vivo gene transfer support the conclusion that $\mathrm{I}_{\text {to1 }}$ plays a crucial role in setting the voltage of the plateau phase. In guinea pig myocytes, $\mathrm{I}_{\mathrm{to} 1}$ suppressed the plateau height in an $\mathrm{I}_{\mathrm{to} 1}$ density-dependent manner, confirming our recent results in myocytes fused with CHO cells expressing Kv4.3 (14). I $_{\text {to1 }}$ reduction in Kv4.3-W362F-infected rat myocytes exhibited the opposite effect, leading to an elevation of the plateau phase compared with wild-type cells. An increase of the plateau voltage has been previously related to low $\mathrm{I}_{\mathrm{to1}}$ density in failing compared with nonfailing canine myocytes (6) and in human subendocardial compared with subepicardial ventriculocytes (27). In addition to a reduction of the peak transient outward current, suppression of the maintained outward current component may have contributed to the changes in the plateau level in Kv4.3-W362F-infected rat myocytes in the present study.

Except for 1 myocyte each, we did not observe a typical notch-and-dome shape of the action potential in Kv4.3-infected guinea pig cells or Kv4.3-W362F-infected rat ventriculocytes. These results support the notion (14) that in addition to $\mathrm{I}_{\mathrm{to} 1}$ density, the balance of other re- and depolarizing currents is crucial for the morphology of phase 1 repolarization and the presence or absence of a notch and dome.

Adenovirus-mediated introduction of $\mathrm{I}_{\mathrm{to} 1}$ into guinea pig myocytes progressively abbreviated APD, whereas $\mathrm{I}_{\mathrm{to} 1}$ suppression in rat myocytes prolonged the action potential. Because $\mathrm{I}_{\mathrm{to} 1}$ rapidly inactivates, transient outward current size must affect APD by influencing the early trajectory of repolarization rather than by a direct contribution to late repolarizing currents $(14,27,33)$. In other tissues or species, it might be possible that $\mathrm{I}_{\mathrm{to} 1}$ suppression results in action potential shortening if the balance of other inward and outward currents were altered in a different fashion. Given that late repolarization was also delayed in $\mathrm{Kv} 4.2$ dominant-negative mice despite an upregulation of a noninactivating outward current (18), it is unlikely that prolongation of the APD in Kv4.3W362F-infected rat ventriculocytes in the present study can simply be attributed to a reduction of the maintained outward current component.

Tachyarrhythmias are a major cause for sudden cardiac death in patients with congestive heart failure (34, 35). Previously, it has been shown that myocytes from dogs with pacing-induced heart failure are more susceptible to EADs compared with nonfailing cells, which led to the hypothesis that malignant arrhythmias in heart failure may arise from abnormalities in repolarization $(36,37)$. Block of $\mathrm{I}_{\text {to1 }}$ by 4-aminopyridine 

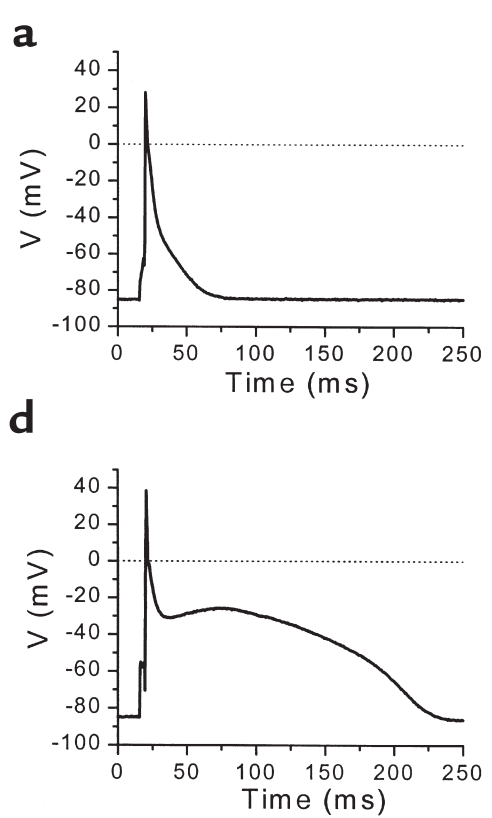

b

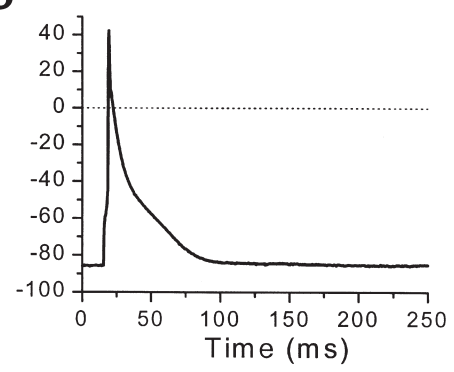

e

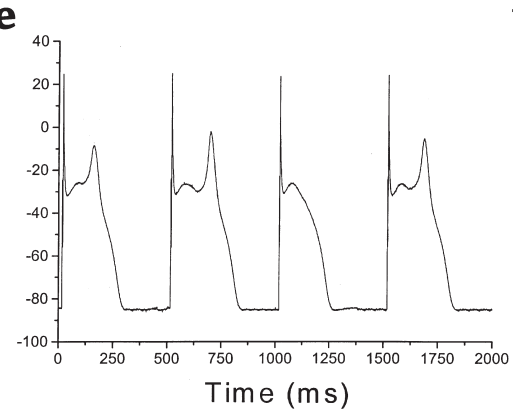

C
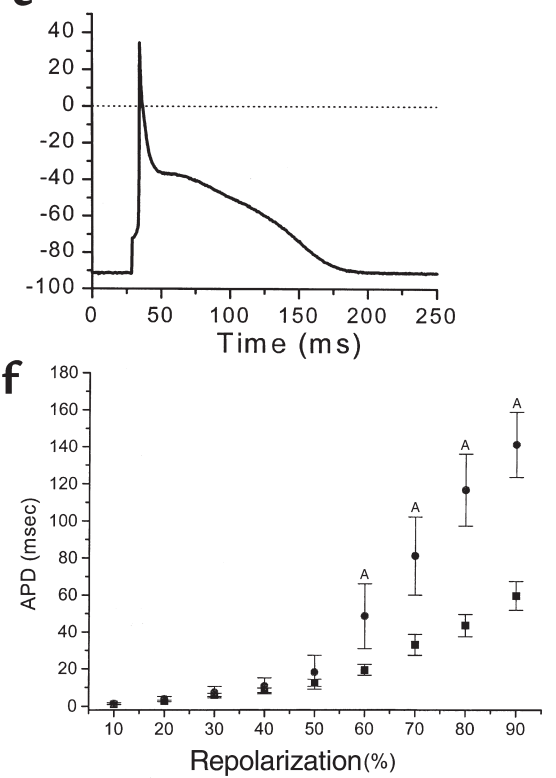

\section{Figure 6}

Action potentials of rat cardiomyocytes were prolonged by dominant-negative in vivo $I_{\text {to } 1}$ suppression. Infection of rat myocytes with AdE8I-Kv4.3W362F substantially changed the action potential waveform ( $\mathbf{c}$ and $\mathbf{d}$ ) compared with noninfected cells ( $\mathbf{a}$ and $\mathbf{b}$ ), resulting in an elevation of the plateau phase and prolongation of the overall APD. In 1 rat myocyte, $I_{\text {to1 }}$ reduction led to a notch-anddome-shaped action potential waveform (d) and to frequent EADs (e). Mean APDs of wild-type (closed squares; $n=10)$ and Kv4.3W362F-infected myocytes (closed circles; $n=7$ ) plotted as a function of the percentage of repolarization $(\mathbf{f})$ demonstrate that $\mathrm{I}_{\text {to } 1}$ suppression did not exhibit a significant effect on the APD at $10-50 \%$ repolarization, but significantly prolonged the $\mathrm{APD}_{60}$ to $\mathrm{APD}_{90}$. Values marked by $\left({ }^{A}\right)$ are significant $(P<0.05)$.

induced frequent EADs in canine M-cells, although nonspecific drug effects on other repolarizing potassium currents may also have contributed to these effects (33). In the present study, we recorded frequent EADs in $1 \mathrm{Kv} 4.3 \mathrm{~W} 362 \mathrm{~F}$-infected rat myocyte; although this was just a single observation, it demonstrates a possible arrhythmogenic mechanism of $\mathrm{I}_{\mathrm{to1}}$ reduction.

ECG recordings from rats injected with the Kv4.3 dominant-negative construct at a single site in the anterior wall of the left ventricle revealed no QT prolongation, presumably because the area of infected myocardium was too small to influence the surface ECG. However, by purposely increasing the number of injection sites, a more widespread adenovirus infection was obtained and the surface ECG was prolonged. Kv4.x suppression in rats resulted in a significant prolongation of the QT interval 72 hours after intramyocardial injection compared with immediate postoperative recordings reflecting the marked prolongation of ventricular action potentials in vivo. These ECG findings demonstrate for the first time, to our knowledge, that somatic gene transfer can be used to create gene-specific animal models of the LQT syndrome. This technology can be applied to larger animals with more humanlike action potentials and also eliminates adaptive changes that can occur during development with transgenic models (18). This targeted reduction in the current of choice obviates the confounding nonspecific effects that can occur in drug-induced models of the LQT syndrome.

Although we used an inducible vector system, the present study was not designed to take full advantage of its potential utility. Two features of the system promise to a

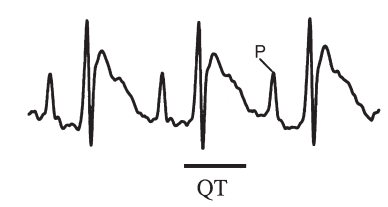

b

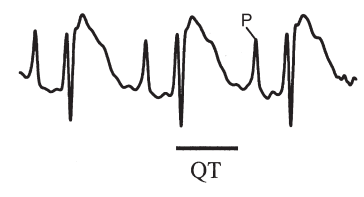

c

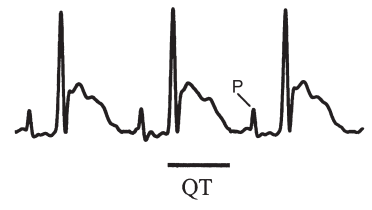

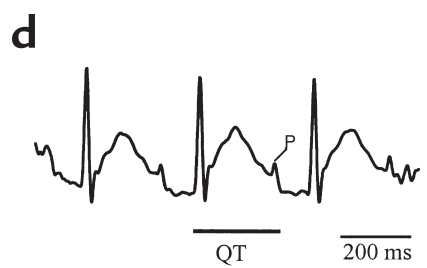

\section{Figure 7}

More widespread infection with AdKv4.3W362F leads to a prolongation of the QT interval. ECG recordings were performed after widespread infection with Kv4.3-W362F or GFP (controls). ECGs revealed a $29.8 \pm 6.0 \%$ prolongation of the QT interval $(P=0.02 ; n=3)$ in Kv4.3-W362F-infected rats 72 hours after intramyocardial injection (d; note the extension of the QT interval into the P wave) compared with immediate postoperative recordings (c), consistent with the marked prolongation of ventricular action potentials. No change in the QT interval was observed in GFP-infected control animals postoperatively (a) compared with 72 hours after infection (b). 
be advantageous in future applications. First, constitutive expression of some ion channel genes results in cytotoxic effects, which prevent the generation of high titer viral stocks (38). Second, inducible vector systems enable the titration of transgene expression levels that may enable precise control of the level of ion channel expression and, therefore, of the magnitude of the effect. To accomplish this, we created a hybrid ecdysone receptor that allowed in vivo induction in the presence of physiological levels of retinoid $\mathrm{X}$ receptor (17), thus also reducing the potential for nonspecific effects of RxR overexpression (39). Effective $\mathrm{I}_{\text {to } 1}$ expression demonstrated that this modified ecdysone system is sufficient not only for inducible in vivo expression of sensitive reporter genes like luciferase, but also for expression of phenotype-altering proteins such as ion channels. Therefore, this hybrid ecdysone system represents a valuable and generalizable tool to probe the function of other proteins in vivo.

\section{Acknowledgments}

We thank A. Ohler for help in confocal imaging. This study was supported by a grant from the National Institutes of Health (P50 HL52370 to E. Marbán), a Research Career Development Award from the CARE Foundation (to D.C. Johns), and a fellowship from the Deutsche Forschungs gemeinschaft (to U.C. Hoppe). E. Marbán is the recipient of the Michel Mirowski, M.D. Professorship in Cardiology of The Johns Hopkins University.

1. CONSENSUS Trail Study Group. 1987. Effects of enalapril on mortality in severe congestive heart failure: results of the Cooperative North Scandinavian Enalapril Survival Study (CONSENSUS). N. Engl. J. Med. 316:1429-1435.

2. SOLVD Investigators. 1991. Effect of enalapril on survival in patients with reduced left ventricular ejection fractions and congestive heart failure. N. Engl. J. Med. 325:293-302.

3. CIBIS Investigators and Committees. 1994. A randomized trial of betablockade in heart failure. The Cardiac Insufficiency Bisoprolol Study (CIBIS). Circulation. 90:1765-1773.

4. Torp-Pedersen, C., et al. 1999. Dofetilide in patients with congestive heart failure and left ventricular dysfunction. Danish Investigations of Arrhythmia and Mortality on Dofetilide Study Group. N. Engl. J. Med. 341:857-865.

5. Beuckelmann, D.J., Näbauer, M., and Erdmann, E. 1993. Alterations of $\mathrm{K}^{+}$currents in isolated human ventricular myocytes from patients with terminal heart failure. Circ. Res. 73:379-385.

6. Kääb, S., et al. 1996. Ionic mechanism of action potential prolongation in ventricular myocytes from dogs with pacing-induced heart failure. Circ. Res. 78:262-273.

7. Rozanski, G.J., Xu, Z., Zhang, K., and Patel, K.P. 1998. Altered K+ current of ventricular myocytes in rats with chronic myocardial infarction. Am. J. Physiol. 274:H259-H265.

8. Dixon, J.E., and McKinnon, D. 1994. Quantitative analysis of potassium channel mRNA expression in atrial and ventricular muscle of rats. Circ. Res. 75:252-260.

9. Dixon, J.E., et al. 1996. Role of the Kv4.3 $\mathrm{K}^{+}$channel in ventricular muscle. A molecular correlate for the transient outward current. Circ. Res. 79:659-668.

10. Johns, D.C., Nuss, H.B., and Marban, E. 1997. Suppression of neuronal and cardiac transient outward currents by viral gene transfer of dominant-negative Kv4.2 constructs. J. Biol. Chem. 272:31598-31603.

11. Fiset, C., Clark, R.B., Shimoni, Y., and Giles, W.R. 1997. Shal-type channels contribute to the $\mathrm{Ca}^{2+}$-independent transient outward $\mathrm{K}^{+}$current in rat ventricle. J. Physiol. 500:51-64.

12. Kääb, S., et al. 1998. Molecular basis of transient outward potassium current downregulation in human heart failure. A decrease in Kv4.3 mRNA correlates with a reduction in current density. Circulation. 98:1383-1393.
13. Mitcheson, J.S., Hancox, J.C., and Levi, A.J. 1998. Cultured adult cardiac myocytes: future applications, culture methods, morphological and electrophysiological properties. Cardiovasc. Res. 39:280-300.

14. Hoppe, U.C., Johns, D.C., Marbán, E., and O’Rourke, B. 1999. Manipulation of cellular excitability by cell fusion: effects of rapid introduction of transient outward $\mathrm{K}+$ current on the guinea pig action potential. Circ. Res. 84:964-972.

15. Inoue, M., and Imanaga, I. 1993. Masking of A-type $\mathrm{K}^{+}$channel in guinea pig cardiac cells by extracellular $\mathrm{Ca}^{2+}$. Am. J. Physiol. 264:C1434-1438.

16. Johns, D.C., Marx, R., Mains, R.E., O’Rourke, B., and Marbán, E. 1999. Inducible genetic suppression of neuronal excitability. J. Neurosci. 19:1691-1697.

17. Hoppe, U.C., Marbán, E., and Johns, D.C. 2000. Adenovirus-mediated inducible gene expression in-vivo by a hybrid ecdysone receptor. $\mathrm{Mol}$. Ther. 1:159-164

18. Barry, D.M., Xu, H., Schuessler, R.B., and Nerbonne, J.M. 1998. Functional knockout of the transient outward current, long-QT syndrome, and cardiac remodeling in mice expressing a dominant-negative Kv4 alpha subunit. Circ. Res. 83:560-567.

19. Hardy, S., Kitamura, M., Harris-Stansil, T., Dai, Y., and Phipps, M.L. 1997. Construction of adenovirus vectors through Cre-lox recombination. J. Virol. 71:1842-1849.

20. Quinones, M.J., et al. 1996. Avoidance of immune response prolongs expression of genes delivered to the adult rat myocardium by replicationdefective adenovirus. Circulation. 94:1394-1401.

21. Dhadialla, T.S., Carlson, G.R., and Le, D.P. 1998. New insecticides with ecdysteroidal and juvenile hormone activity. Annu. Rev. Entomol. 43:545-569.

22. Mitra, R., and Morad, M. 1986. Two types of calcium channels in guinea pig ventricular myocytes. Proc. Natl. Acad. Sci. USA. 83:5340-5344.

23. Hamill, O.P., Marty, A., Neher, E., Sakmann, B., and Sigworth, F.J. 1981. Improved patch-clamp techniques for high-resolution current recording from cells and cell-free membrane patches. Pflugers Arch. 391:85-100.

24. Neher, E. 1992. Correction for liquid junction potentials in patch clamp experiments. Methods Enzymol. 207:123-131.

25. Johns, D.C., et al. 1995. Adenovirus-mediated expression of a voltagegated potassium channel in vitro (rat cardiac myocytes) and in vivo (rat liver). J. Clin. Invest. 95:1152-1158.

26. No, D., Yao, T.P., and Evans, R.M. 1996. Ecdysone-inducible gene expression in mammalian cells and transgenic mice. Proc. Natl. Acad. Sci. USA. 93:3346-3351.

27. Näbauer, M., Beuckelmann, D.J., Uberfuhr, P., and Steinbeck, G. 1996. Regional differences in current density and rate-dependent properties of the transient outward current in subepicardial and subendocardial myocytes of human left ventricle. Circulation. 93:168-177.

28. Baldwin, T.J., Tsaur, M.L., Lopez, G.A., Jan, Y.N., and Jan, L.Y. 1991. Characterization of a mammalian cDNA for an inactivating voltage-sensitive K+ channel. Neuron. 7:471-483.

29. Hayes, E., Pugsley, M.K., Penz, W.P., Adaikan, G., and Walker, M.J. 1994 Relationship between QaT and RR intervals in rats, guinea pigs, rabbits, and primates. J. Pharmacol. Toxicol. Methods. 32:201-207.

30. Rees, S.A., and Curtis, M.J. 1993. Tacrine inhibits ventricular fibrillation induced by ischaemia and reperfusion and widens QT interval in rat. Cardiovasc. Res. 27:453-458.

31. Tu, L., et al. 1996. Voltage-gated $\mathrm{K}+$ channels contain multiple intersubunit association sites. J. Biol. Chem. 271:18904-18911.

32. Clark, R.B., Bouchard, R.A., Salinas-Stefanon, E., Sanchez-Chapula, J., and Giles, W.R. 1993. Heterogeneity of action potential waveforms and potassium currents in rat ventricle. Cardiovasc. Res. 27:1795-1799.

33. Antzelevitch, C., et al. 1991. Heterogeneity within the ventricular wall. Electrophysiology and pharmacology of epicardial, endocardial, and $\mathrm{M}$ cells. Circ. Res. 69:1427-1449.

34. Liberthson, R.R., Nagel, E.L., Hirschman, J.C., and Nussenfeld, S.R. 1974 Pre-hospital ventricular fibrillation: prognosis and follow-up course. $N$. Engl. J. Med. 219:317-321.

35. Luu, M., Stevenson, W.G., Stevenson, L.W., Baron, K., and Walden, J. 1989. Diverse mechanisms of unexpected cardiac arrest in advanced heart failure. Circulation. 80:1675-1680.

36. Nuss, H.B., Kaab, S., Kass, D.A., Tomaselli, G.F., and Marbán, E. 1999. Cellular basis of ventricular arrhythmias and abnormal automaticity in heart failure. Am. J. Physiol. 277:H80-H91.

37. Marbán, E. 1999. Heart failure: the electrophysiologic connection. J. Cardiovasc. Electrophysiol. 10:1425-1428.

38. Johns, D.C. 1999. Genetic modification of cellular excitability. Dissertation. Johns Hopkins University School of Medicine. Baltimore, MD. 1-197.

39. Subbarayan, V., et al. 2000. RXRalpha overexpression in cardiomyocytes causes dilated cardiomyopathy but fails to rescue myocardial hypoplasia in RXRalpha-null fetuses. J. Clin. Invest. 105:387-394. 